\title{
The Invisible Risks: "Musculoskeletal Symptoms Among Medical and Dental Professions Concerning Work Posture."
}

\author{
K Srinivasan*, Varsha Modipalle and R Venkata Subramanian \\ Department of Pedodontic and Preventive Dentistry, CKS Theja Institute of Dental Sciences and Research, Chadalawada Nagar, Renigunta \\ Road, Tirupati, India \\ *Corresponding Author: K Srinivasan, Department of Pedodontic and Preventive Dentistry, CKS Theja Institute of Dental Sciences and \\ Research, Chadalawada Nagar, Renigunta Road, Tirupati, India.
}

Received: September 19, 2019; Published: October 24, 2019

DOI: $10.31080 /$ ASDS.2019.03.0676

\begin{abstract}
Background: Musculoskeletal disorders (MSD) are one of the most common occupational hazards associated with wide ranges of occupations. Inappropriate posture may increase fatigue, decrease efficiency, and eventually lead to injuries.

Aim: The purpose was to assess the Ergonomic risk level in Medicine and Dentistry, which may contribute to the manifestation of musculoskeletal disorders (MSD).

Methods and Materials: A cross-sectional, descriptive study in which a self-administered questionnaire (Standardized Nordic questionnaire) was used to access the relationship between anthropometric data and the risk of work-related musculoskeletal disorders (WRMDs) among the Dental and Medical professionals.

A self-reported questionnaire was distributed to a random sample of 300 professionals. The recorded data were analyzed with SPSS 21. p- value $<0.05$ was considered to be statistically significant.

Results: A total of 257 questionnaires were completed and returned. Of the respondents, 141 (54.8\%) were males, and 116 (45.1\%) were females. The mean age of respondents was 33 with SD 6.01. Prevalence of MSD during the past six months was lower back among both Dental and Medical professionals, 36.5\% and 30.1\% respectively. Results were statistically significant done.

Conclusion: The results suggested that the prevalence of musculoskeletal symptoms among Dentists was high. MSD remains a major occupational health problem for both professionals with the most prevalent issue of the lower back and pain neck. It is essential for promoting the occupational health and prevention programs regarding Ergonomic postures which must be acquired by the Medical and Dental profession during their clinical practices.
\end{abstract}

Keywords: Musculoskeletal Disorders; Ergonomics; Dentistry; Medical; Survey

\section{Introduction}

Hospitals are complex systems relying on an incredibly diverse group of occupations, many of which are physically demanding [1]. Working with equipment that's comfortable to use, and having adequately designed work areas relate directly to safety, unusual in the prevention of work-related musculoskeletal disorders. They reduce muscle fatigue in workers that can lead to injuries and illnesses [1].

Today, Musculoskeletal disorders (MSDs) are one of the most common occupational diseases and causes of disability in industrialized countries and developing countries. The most important causes of the dysfunction can be awkward postures, 
repetitive and rapid movements, excessive force, Psychological factors, genetic and generally inappropriate body condition, and work stations [2].

Musculoskeletal disorder is characterized by the presence of discomfort, disability or persistent pain in the joints, muscles, tendons, and other soft parts [3].

Musculoskeletal disorders and stress-related illnesses were the two most important factors that influenced premature retirement $[4]$.

According to scientific literature, the Dental professions are one of the most susceptible to occupational diseases [5].

Back pain is the most common complaint followed by neck pain and shoulder pain, though they all are usually mild. Most Dentists today work in the sitting position and treat the patient in the supine position. Being seated made little difference in how frequently the operator experiences pain. When operators sit, pain occurs not only in their back but also in their neck, shoulders, and arms. While the occasional backache or neck aches is not a cause for alarm, if regularly occurring pain or discomfort which is ignored, the cumulative Physiological damage can lead to an injury or a careerending disability [6].

In Greek, "Ergo," means work and, "Nomos," means natural laws or systems. Ergonomics, therefore, is an applied science concerned with designing products and procedures for maximum efficiency and safety [7].

It is also a study of the relationship among the personnel, equipment, and environment in the work area [8].

Proper Ergonomic design is necessary to prevent repetitive strain injuries, which can develop over time and can lead to longterm disability. Ergonomics is concerned with the 'fit' between people and their work. It takes account of the worker's capabilities and limitations in seeking to ensure that tasks, equipment, information, and the environment suit each worker [9].

Common injuries due to poor Ergonomics at work stations usually affect back, neck and upper limb and are mainly caused by repetition and overstrain at tendons and joints, unbalanced and prolonged postures, chronic inflammation and weakness [10].
Anthropometry can be defined as the study which deals with body dimensions, i.e., body size, shape, strength, and working capacity [11] for design purposes [12] and body composition [13].

This study was planned and carried out to find the prevalence of MSD among Medical and Dental professionals at their workplaces using DMAIC technique which involves defining, measuring, analyzing and then improving by the implementation of methods to control the problem.

\section{Research methodology}

\section{Objectives}

1. To determine the prevalence of musculoskeletal pain among private Medical and Dental practitioners.

2. To determine risk factors associated with the development of musculoskeletal pain among private Medical and Dental practitioners.

\section{Study design}

During 2019 a descriptive-analytical, cross-sectional study was planned and carried out to determine musculoskeletal work-related pain and Anthropometric measurement of 18 body dimensions.

The pool of participants for the feasibility study was carried out on all Medical and Dental professionals who worked in private and public offices in the city of Vellore and Tirupati. The selection was made by a simple random sampling method Ethical clearance was taken from the institutional ethical committee for the commencement of the study. The purpose of the study was explained to the participants, and informed consent was obtained.

The data collection was done over three months, from July to September 2019.

\section{Study Settings}

The study was planned in three phases.

a) The first part was a self-administered questionnaire about the respondents' demographic information.

b) In the second part, Basic Anthropometric data was collected from each participant.

c) In the third part, the Ergonomic technique during their operation questionnaire. 
Inclusion criteria

a) General practitioners and specialists between the ages of 25-45 years.

b) No history of inflammatory and degenerative Rheumatic diseases.

c) No known motor deficiencies in the upper and lower back.

d) Right-handed Dentists and Medical professionals.

Exclusive criteria

a) History of fractures or muscular tear of lower members, those who played sports at least thrice a week.

b) No known Orthopedics or Neurological disorder.

c) Participant suffering from previously diagnosed Scoliosis, Chronic lumbar pain, -those who also a history of MS surgeries, and current pregnancy.

\section{Subject recruitment}

Tools used for data collection designed by the researcher based on information from the literature review and revised by subject area experts. Validity; determined by Experts. Reliability assessed twice by applying the tools on 20 subjects who excluded from the study sample (pilot study). The reliability of the questionnaire was determined by using Cronbach's alpha coefficient test value degree 0.92 .

Anthropometric parameters for the population group for a $95 \%$ confidence interval for the 5 th, 50 th, and 95 th percentiles were obtained using formulae:

$\mathrm{n} \geq(3.006 \times \mathrm{CV} / \alpha) 2$

Where,

- $\mathrm{n}$ : sample size,

- $\quad \mathrm{CV}$ : coefficient of variation

- $\alpha$ : the percentage of relative accuracy desired.

A total of 300 (age range: 25 - 40 years; 141 male, 116 female Dentists and Medical professionals received the questionnaire on the topic of musculoskeletal work-related pain in their offices.

However, from the total participants, $85.6 \%(n=25)$ accepted to sign the consent form and admitted to this study. The main reason to refuse contribution was due to being observed by another person during his or her task and lack of time.
Questionnaire and data collection

The questionnaire used in this study was adopted and modified from previous studies.

\section{Data collection}

The study was carried out in three phases. The first part was a self-administered questionnaire about the respondents' demographic information, including age, sex, time of post, their principal task, and job satisfaction. Job satisfaction was evaluated based on the comfort of the work environment, lighting, staff, unit equipment, and Dentists and Medical professional' working chair using an open and closed-end Questionnaire.

In the second part, Basic Anthropometric data were collected from each participant recognized by the Nordic Musculoskeletal Questionnaire (NMQ) [14] suitable for application in workplaces. The questionnaire included six body areas, including the neckshoulders, elbows-wrist, lower back, hip- thighs, knees, and anklesfeet. Musculoskeletal complaints were defined as pain perceived in the last six months experienced as ache, discomfort, and numbness, trouble over the previous seven days and preventing activity during last six months.

In the third part- Anthropometric profile: All measurement was taken twice by a trained researcher which was measured according to Abeysekera and Pheasant and Haslegrave [15] as displayed in Figure 1. A traditional Anthropometer (Galaxy Informatics India), a measuring tape (Freemans Levo Steel Measuring tape), and a Weight measured by using a pre-calibrated Digital Weight scale (Granny Smith Bolt Analog Weight Machine.). Height measured by using a portable Stadiometer (Seca 213).

This Ergonomic technique evaluates people's exposures to postures, forces, and muscle activities that contribute to Repetitive Strain Injuries; the postures of the neck, trunk, and upper limbs.

Thus, in all the three phases, the musculoskeletal problem was defined, measured, and analyzed using the first three steps of DMAIC methodology. After three months, subjects were again approached and given a questionnaire about the MSD.

\section{Statistical analysis}

Data were anonymously coded and entered into a spreadsheet program before being analyzed using the Statistical Package for the Social Sciences-SPSS 21(Japan IBM Co., Ltd, Tokyo, Japan)). 
The Invisible Risks: “Musculoskeletal Symptoms Among Medical and Dental Professions Concerning Work Posture."

Appropriate statistical methods such as frequency, descriptive measures, independent t-test, and Mann Whitney test were performed in a proper context. A 95\% Confidence Level was used, and a p-value of less than or equal to 0.05 was considered statistically significant.

\section{Results}

Statistical analysis using analysis of variance (ANOVA) was used to identify any significant differences among different populations at $\mathrm{p}=0.05$.

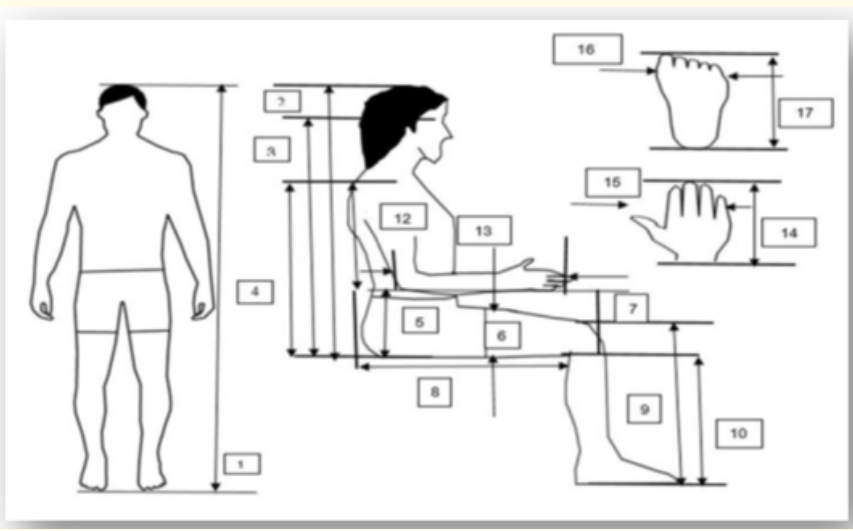

\begin{tabular}{|c|c|c|}
\hline \multicolumn{3}{|r|}{ Figure.1.Anthropometric measured position } \\
\hline $\begin{array}{c}\text { Ref } \\
\text { no }\end{array}$ & Measurement & Description \\
\hline 1. & Stature. & The vertical distance from the floor to the vertex. \\
\hline 2. & Sitting Height. & Vertical distance from the sitting surface to the vertex. \\
\hline 3. & Sitting Eye Height. & Vertical distance from the sitting surface to the inner corner of the eye. \\
\hline 4. & Sitting Shoulder Height. & Vertical distance from the sitting surface to the acromion. \\
\hline 5. & Sitting Elbow height. & Vertical distance from the sitting surface to the underside of the elbow. \\
\hline 9. & Knee Height. & Vertical distance from the floor to the upper surface of the knee. \\
\hline 12. & Shoulder-Elbow Length. & Distance from the acromion to underside of the elbow in a sitting position. \\
\hline 13. & Elbow Fingertip Length. & Distance from the back of the elbow to the tip of the middle finger in a standard sitting position. \\
\hline 15. & Hand Breadth. & Maximum breath across the palm of the hand. \\
\hline & & $\begin{array}{l}\text { Forward bending of the head }\left(<20^{9}\right) . \\
\text { Craniovertebral angle measurement. }\end{array}$ \\
\hline & & $\begin{array}{l}\text { Trunk rotation }\left(<20^{\circ}\right) \\
\text { The individual is asked to rotate toward the forward leg (right leg forward, rotation to the } \\
\text { right). }\end{array}$ \\
\hline & & $\begin{array}{l}\text { Trunk sideward inclination }(<109) \\
\text { Trunk forward inclination was measured as the angle between the long axis of the trunk } \\
\text { segment and the } z \text {-axis in the } x-z \text { plane }\end{array}$ \\
\hline & & BMI $=$ weight $(\mathrm{kg}) /[\text { height }(\mathrm{m})]^{2}$ \\
\hline
\end{tabular}

Figure 1 
The Invisible Risks: "Musculoskeletal Symptoms Among Medical and Dental Professions Concerning Work Posture."

\section{Demographical and occupational data}

$70.6 \%$ of Dentists $(\mathrm{n}=123)$ and $67.4 \%(\mathrm{n}=56)$ Medical professionals were aware of proper Ergonomics (Table 1, Graph.1), whereas 29\% $(n=51)$ and 32\% $(n=27)$ were not aware of Ergonomics and its implications in Dentistry and Medicine respectively. They claimed that they have never focused on excellent Ergonomics in their practice.
The mean age and mean was $33.0 \pm 6.01$. Half of the subjects (50\%) reported more significant than 4 hours of busy daily work. Table 1 provides data on demographics, working position, exercising frequency, and impact of neck/back pain on practice.

\begin{tabular}{|c|c|c|c|c|c|}
\hline \multicolumn{6}{|l|}{ Individual Scenario. } \\
\hline Variables & \multicolumn{3}{|c|}{ Respondents } & Responses (n) & Frequency (\%) \\
\hline \multicolumn{4}{|l|}{ Total number of respondents. } & 257 & 85.6 \\
\hline \multicolumn{4}{|l|}{ Age. [Years mean (SD)]. } & \multicolumn{2}{|c|}{$33 \pm 6.01$} \\
\hline \multirow{2}{*}{ Gender. } & \multicolumn{3}{|c|}{ Male. } & 141 & 54.8 \\
\hline & \multicolumn{3}{|c|}{ Female. } & 116 & 45.1 \\
\hline \multirow[t]{6}{*}{ Qualification. } & \multirow[t]{4}{*}{ Dental } & UG Student. & 47 & \multirow[b]{4}{*}{174} & \multirow[b]{4}{*}{67.7} \\
\hline & & PG Student. & 41 & & \\
\hline & & General Practitioners. & 48 & & \\
\hline & & Specialist. & 38 & & \\
\hline & \multirow[t]{2}{*}{ Medical } & General Practitioners. & 48 & \multirow[b]{2}{*}{83} & \multirow[t]{2}{*}{32.2} \\
\hline & & Specialist & 35 & & \\
\hline \multirow[t]{2}{*}{ Nature of practice. } & \multicolumn{3}{|c|}{ Full time. } & 139 & 54.0 \\
\hline & \multicolumn{3}{|c|}{ Part-time. } & 118 & 45.9 \\
\hline \multirow[t]{2}{*}{ The number of patients seen per day. } & \multicolumn{3}{|c|}{$\leq 20$} & 197 & 76.6 \\
\hline & \multicolumn{3}{|c|}{$21-40$} & 60 & 23.3 \\
\hline \multirow[t]{3}{*}{ Associate Satisfaction. } & \multicolumn{3}{|c|}{ Yes. } & 165 & 64.2 \\
\hline & \multicolumn{3}{|c|}{ No. } & 31 & 12.0 \\
\hline & \multicolumn{3}{|c|}{ Rarely. } & 61 & 23.7 \\
\hline \multirow[t]{3}{*}{ Environmental satisfaction. } & \multicolumn{3}{|c|}{ Yes. } & 207 & 80.5 \\
\hline & \multicolumn{3}{|c|}{ No. } & 33 & 12.8 \\
\hline & \multicolumn{3}{|c|}{ Rarely. } & 17 & 6.6 \\
\hline \multirow[t]{3}{*}{ Tabor satisfaction. } & \multicolumn{3}{|c|}{ Yes. } & 211 & 82.1 \\
\hline & \multicolumn{3}{|c|}{ No. } & 38 & 14.7 \\
\hline & & Rarely. & & 8 & 3.11 \\
\hline Aware of proper Ergonomics and its & Medical practice. & Yes. & & 123 & 70.6 \\
\hline implications. & & No. & 51 & 29.3 & \\
\hline & Dental practice. & Yes. & & 56 & 67.4 \\
\hline & & No. & 27 & 32.5 & \\
\hline
\end{tabular}


The Invisible Risks: "Musculoskeletal Symptoms Among Medical and Dental Professions Concerning Work Posture."

\begin{tabular}{|c|c|c|c|}
\hline \multirow[t]{2}{*}{ Decreased grip strength. } & Yes. & 165 & 64.2 \\
\hline & No. & 92 & 35.7 \\
\hline \multirow[t]{2}{*}{ Decreased range of motion. } & Yes. & 184 & 71.5 \\
\hline & No. & 73 & 28.4 \\
\hline \multirow{3}{*}{$\begin{array}{l}\text { Interruption of patient treatment due } \\
\text { to neck/back pain. }\end{array}$} & Never. & 192 & 74.7 \\
\hline & Rarely. & 39 & 15.1 \\
\hline & Occasionally. & 26 & 10.1 \\
\hline \multirow{2}{*}{$\begin{array}{l}\text { Participant suffering from Musculo- } \\
\text { skeletal problems. }\end{array}$} & Yes. & 189 & 73.5 \\
\hline & No. & 68 & 26.4 \\
\hline \multirow{7}{*}{$\begin{array}{l}\text { Did you administered Medicines for } \\
\text { relief or consulted the Orthopaedi- } \\
\text { cians for discomfort. }\end{array}$} & NSAID & 77 & $29.9 \%$ \\
\hline & Correct posture & 46 & $17.8 \%$ \\
\hline & Muscle relaxing exercise & 68 & $26.4 \%$ \\
\hline & Reduce working hours. & 32 & $12.4 \%$ \\
\hline & Seek specialist opinion. & 27 & $10.5 \%$ \\
\hline & To abstain from work. & 26 & $10.1 \%$ \\
\hline & None of The Above & 27 & 10.5 \\
\hline
\end{tabular}

Table 1: Social -Demographic Variables Of Respondents $(n=300)$.

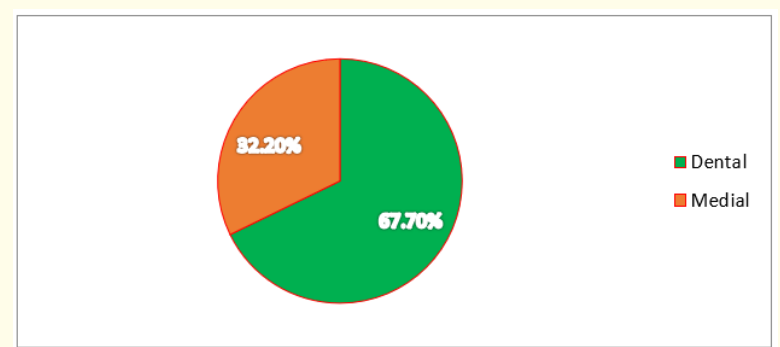

Graph 1: Number of participants (n=257).

\section{Frequency and impact of musculoskeletal complaints}

Detailed distributions of the individual categories of claims by working position are given in Table 2, 3, Graph.2. Musculoskeletal pain was quite common among both professionals with a prevalence of $73.5 \%(n=189)$. Moreover, $10.5 \%(n=27)$ of professionals had sought Medical help for musculoskeletal complaints. Among the categories, lower back pain was the most frequent among the Dentist (36.7\%), neck and shoulder pain
(25.3\%), among the Medical profession. On the other hand, 74.7\% ( $n=192$ ) of the subjects had never experienced musculoskeletal symptoms necessitating the interruption of the patient treatment Majority of the Dentists and Medical reported that they preferred sitting, 59.1\% $(n=103)$ and $65.06 \%(n=54)$ position respectively when working.

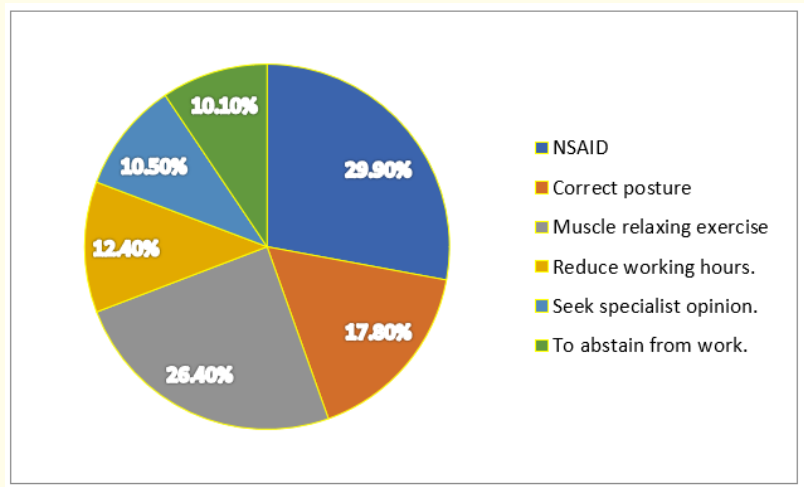

Graph 2: MDS- Preventive measures (n=257). 
The Invisible Risks: “Musculoskeletal Symptoms Among Medical and Dental Professions Concerning Work Posture."

\begin{tabular}{|c|c|c|c|c|c|c|c|c|c|}
\hline \multicolumn{10}{|l|}{ Individual Scenario } \\
\hline Variables & \multicolumn{6}{|c|}{ Responses n (\%) } & \multicolumn{3}{|l|}{ Inference } \\
\hline \multicolumn{10}{|l|}{ Position in the work } \\
\hline & Pedo & Prostho & $\begin{array}{l}\text { Conserva- } \\
\text { tive \& Endo }\end{array}$ & $\begin{array}{c}\text { Oral } \\
\text { Sugery }\end{array}$ & Perio & Ortho & Mean \pm SD & $\mathrm{Z}$ value & $\mathrm{p}$-value \\
\hline $\begin{array}{l}\text { Sitting Dentistry } \\
103(59.1 \%)\end{array}$ & $10(5.7)$ & $16(9.19)$ & $27(15.5)$ & $17(9.7)$ & $11(6.3)$ & $22(12.6)$ & \multirow[t]{2}{*}{$14.5 \pm 8.9$} & \multirow[t]{2}{*}{17.8} & \multirow[t]{2}{*}{$\mathrm{p}<0.0001 \mathrm{HS}$} \\
\hline $\begin{array}{l}\text { standing Dentistry } \\
71(40.8 \%)\end{array}$ & $6(3.4)$ & $35(20.1)$ & $11(6.3)$ & $8(4.5)$ & $5(2.8)$ & $6(3.4)$ & & & \\
\hline \multicolumn{10}{|l|}{ Vision in the work } \\
\hline $\begin{array}{l}\text { Direct } \\
115(66 \%)\end{array}$ & $15(8.6)$ & $16(9.1)$ & $31(17.8)$ & $18(10.3)$ & $10(5.7)$ & $25(14.3)$ & \multirow[t]{2}{*}{$14.5 \pm 6.8$} & \multirow[t]{2}{*}{23.2} & \multirow[t]{2}{*}{$\mathrm{p}<0.0001 \mathrm{HS}$} \\
\hline $\begin{array}{l}\text { Indirect } \\
59(33.4 \%) \\
\end{array}$ & $9(5.1)$ & $11(6.3)$ & 105.7) & $7(4.02)$ & $12(6.8)$ & $10(5.7)$ & & & \\
\hline \multicolumn{10}{|l|}{ Site of pain } \\
\hline $\begin{array}{l}\text { Neck and Shoulders } \\
27(15.5 \%)\end{array}$ & $5(2.8)$ & $1(0.5)$ & $6(3.4)$ & $4(2.2)$ & $8(4.5)$ & $3(1.7)$ & \multirow{6}{*}{$4.9 \pm 3.6$} & \multirow{6}{*}{45.7} & \multirow{6}{*}{$\mathrm{p}<0.0001 \mathrm{HS}$} \\
\hline $\begin{array}{l}\text { Elbows and Wrists } 6 \\
(3.4 \%)\end{array}$ & 0 & 0 & $2(1.1)$ & $1(0.5)$ & $2(1.1)$ & $1(0.5)$ & & & \\
\hline $\begin{array}{l}\text { Low back } \\
64(36.7 \%)\end{array}$ & $9(5.1)$ & $7(4.02)$ & $15(8.6)$ & $8(4.5)$ & $13(7.4)$ & $12(6.8)$ & & & \\
\hline $\begin{array}{l}\text { One or both Hips and } \\
\text { Thighs } \\
17(11.8 \%)\end{array}$ & $1(0.5)$ & $1(0.5)$ & $6(3.4)$ & $2(1.1)$ & $4(2.2)$ & $3(1.7)$ & & & \\
\hline $\begin{array}{l}\text { One or both Knees } \\
22(12.6 \%)\end{array}$ & $3(1.7)$ & $2(1.1)$ & $7(4.02)$ & $3(1.7)$ & $4(2.2)$ & $3(1.7)$ & & & \\
\hline $\begin{array}{l}\text { One or both Ankles/ } \\
\text { Feet } \\
38(21.8 \%)\end{array}$ & $4(2.2)$ & $4(2.2)$ & $10(5.7)$ & $3(1.7)$ & $8(4.5)$ & $9(5.1)$ & & & \\
\hline
\end{tabular}


The Invisible Risks: “Musculoskeletal Symptoms Among Medical and Dental Professions Concerning Work Posture."

\begin{tabular}{|c|c|c|c|c|c|c|c|c|c|}
\hline \multicolumn{10}{|c|}{ The average duration of work per day. (in hours) } \\
\hline & $\begin{array}{c}\text { Neck } \\
\text { also, Shoul- } \\
\text { ders }\end{array}$ & $\begin{array}{l}\text { Elbows } \\
\text { and } \\
\text { Wrists }\end{array}$ & Low back & $\begin{array}{l}\text { One or } \\
\text { both } \\
\text { Hips and } \\
\text { Thighs }\end{array}$ & $\begin{array}{l}\text { One or } \\
\text { both } \\
\text { Knees }\end{array}$ & $\begin{array}{c}\text { One or } \\
\text { both } \\
\text { Ankles/ } \\
\text { Feet }\end{array}$ & Mean \pm SD & $\begin{array}{c}\mathrm{Z} \\
\text { value }\end{array}$ & p-value \\
\hline $\begin{array}{l}\text { 1-2 hours- } \\
27(15.5 \%)\end{array}$ & $4(2.2)$ & $2(1.1)$ & $3(1.7)$ & $6(3.4)$ & $10(5.7)$ & $2(1.1)$ & \multirow[t]{3}{*}{$9.6 \pm 5.1$} & \multirow{3}{*}{3.1} & \multirow[t]{3}{*}{$\mathrm{p}<0.0001 \mathrm{HS}$} \\
\hline $\begin{array}{l}\text { 2-4 hours- } \\
60(34.4 \%)\end{array}$ & $6(3.4)$ & $7(4.02)$ & $17(9.7)$ & $11(6.2)$ & $8(4.5)$ & $11(6.3)$ & & & \\
\hline $\begin{array}{l}\text { Greater than } 4 \\
\text { hours- } 87(50 \%)\end{array}$ & $17(9.7)$ & $16(9.1)$ & $12(6.8)$ & $\begin{array}{c}19 \\
(10.9)\end{array}$ & $10(5.7)$ & $13(7.4)$ & & & \\
\hline \multicolumn{10}{|l|}{ Reason for pain } \\
\hline \multicolumn{4}{|l|}{ Stress. } & \multicolumn{3}{|c|}{$69(39.6)$} & \multirow{7}{*}{$24.8 \pm 21.5$} & \multirow{7}{*}{6.9} & \multirow{7}{*}{$\mathrm{p}<0.0001 \mathrm{HS}$} \\
\hline \multicolumn{4}{|l|}{ Fatigue. } & \multicolumn{3}{|c|}{$43(24.7)$} & & & \\
\hline \multicolumn{4}{|l|}{ Nervousness. } & \multicolumn{3}{|c|}{$22(12.6)$} & & & \\
\hline \multicolumn{4}{|l|}{ Anxiety. } & \multicolumn{3}{|c|}{$11(6.3)$} & & & \\
\hline \multicolumn{4}{|c|}{ Tension before going to work. } & \multicolumn{3}{|c|}{$6(3.4)$} & & & \\
\hline \multicolumn{4}{|c|}{ Awakening at night. } & \multicolumn{3}{|c|}{$17(9.7)$} & & & \\
\hline \multicolumn{4}{|c|}{ Genetic Predisposition. } & \multicolumn{3}{|c|}{$6(3.4)$} & & & \\
\hline
\end{tabular}

Table 2: Standardized Nordic Questionnaire (Snq) About Pain and Discomfort In Various Locomotor Organs. -Dental (n=174) Statistical Inference: HS-Highly significant SS- Statistical significant.

\begin{tabular}{|c|c|c|c|c|}
\hline \multicolumn{5}{|l|}{ Individual Scenario } \\
\hline Variables & Responses (\%) & Mean \pm SD & $Z$ value & p-value \\
\hline \multicolumn{5}{|l|}{ Position in the work. } \\
\hline Sitting. & $54(65.06)$ & \multirow[b]{2}{*}{$41.5 \pm 12.5$} & \multirow[b]{2}{*}{3.2} & \multirow[t]{2}{*}{$\mathrm{p}<0.0001 \mathrm{HS}$} \\
\hline Standing. & $29(34.9)$ & & & \\
\hline \multicolumn{5}{|l|}{ Site of pain } \\
\hline Neck and Shoulders. & $25(65.06)$ & \multirow{6}{*}{$13.5 \pm 7.4$} & \multirow{6}{*}{9.3} & \multirow{6}{*}{$\mathrm{p}<0.0001 \mathrm{HS}$} \\
\hline Elbows and Wrists. & $5(6.02)$ & & & \\
\hline Low back. & $21(25.3)$ & & & \\
\hline One or both Hips and Thighs. & $7(8.4)$ & & & \\
\hline One or both Knees. & $15(9.6)$ & & & \\
\hline One or both Ankles/Feet. & $8(7.6)$ & & & \\
\hline \multicolumn{5}{|c|}{ The average duration of work per day. (in hours) } \\
\hline $1-2$ hours. & $21(25.3)$ & \multirow{3}{*}{$27.6 \pm 6.2$} & \multirow{3}{*}{8.8} & \multirow[t]{3}{*}{$\mathrm{p}<0.0001 \mathrm{HS}$} \\
\hline 2-4 hours. & $26(31.3)$ & & & \\
\hline Greater than 4 hours. & $36(43.3)$ & & & \\
\hline \multicolumn{5}{|l|}{ Reason for pain } \\
\hline Stress. & $25(30.1)$ & \multirow{7}{*}{$11.8 \pm 6.3$} & \multirow{7}{*}{11.4} & \multirow{7}{*}{$\mathrm{p}<0.0001 \mathrm{HS}$} \\
\hline Fatigue. & $14(16.8)$ & & & \\
\hline Nervousness. & $8(9.6)$ & & & \\
\hline Anxiety. & $7(8.4)$ & & & \\
\hline Tension before going to work. & $9(10.8)$ & & & \\
\hline Awakening at night. & $15(18.07)$ & & & \\
\hline Genetic Predisposition. & $5(6.02)$ & & & \\
\hline
\end{tabular}

Table 3: Standardized Nordic Questionnaire (Snq) About Pain And Discomfort In Various Locomotor Organs.-Medical (n=83) Statistical Inference: HS-Highly significant SS- Statistical significant 
Anthropometric data development and analysis

The descriptive and inferential statistics of the anthropometric characteristics and indices depicted in Table 4, 5, Graph.3, 4. The means of stature, sitting height erect, and body mass of the subjects were $177.9 \pm 5.0,87.2 \pm 4.1,25.0 \pm 0.6$ for males $(n=44)$ and 169.2 $\pm 2.9,22.9 \pm 1.4$ for females respectively.
The standard deviation of the anthropometric dimensions varies from 0.4 to 5.9. The highest St. Dev. of stature indicates that the maximum number of sample deviates from their mean of stature measurement.

This standard representation of the data allows the designers and health practitioners to identify the size range of each measurement.

\begin{tabular}{|c|c|c|c|c|c|c|c|}
\hline \multirow{2}{*}{ Ref no } & \multirow{2}{*}{ Variables } & \multirow{2}{*}{ Mean \pm SD } & \multirow{2}{*}{$\mathrm{Z}$ value } & \multirow{2}{*}{ p- value } & \multicolumn{3}{|c|}{ Percentile } \\
\hline & & & & & $5^{\text {th }}$ & $\mathbf{5 0}^{\text {th }}$ & $95^{\text {th }}$ \\
\hline 1 & Stature. & $177.9 \pm 5.0$ & -26.4 & $\mathrm{P}<0.0001 \mathrm{HS}$ & 173 & 176.5 & 183 \\
\hline 2 & Sitting Height. & $87.2 \pm 4.1$ & -10.3 & $\mathrm{P}<0.0001 \mathrm{HS}$ & 81.7 & 86.6 & 91.4 \\
\hline 3 & Sitting Eye Height. & $74.8 \pm 4.7$ & -6.4 & $\mathrm{p}<0.0001 \mathrm{HS}$ & 69.5 & 73.9 & 74.1 \\
\hline 4 & Sitting Shoulder Height. & $55.4 \pm 3.2$ & -3.5 & $\mathrm{p}<0.0001 \mathrm{HS}$ & 50.1 & 55.6 & 59.1 \\
\hline 5 & Sitting Elbow height. & $19.4 \pm 3.2$ & 7.5 & $\mathrm{p}<0.0001 \mathrm{HS}$ & 14.1 & 19.6 & 24.2 \\
\hline 9 & Knee Height. & $48.8 \pm 4.5$ & -1.0 & $\mathrm{P}=0.281 \mathrm{NS}$ & 41.5 & 48.9 & 55.1 \\
\hline 12 & Shoulder-Elbow Length. & $33.6 \pm 3.7$ & 3.7 & $\mathrm{p}<0.0001 \mathrm{HS}$ & 29.1 & 33.5 & 37.9 \\
\hline 13 & Elbow Fingertip Length. & $48.7 \pm 5.9$ & 0.7 & $\mathrm{p}=0.42 \mathrm{NS}$ & 39.7 & 48.7 & 57.2 \\
\hline 14 & Hand length. & $18.2 \pm 2.3$ & 10.9 & $\mathrm{p}<0.0001 \mathrm{HS}$ & 14.5 & 18.5 & 22.7 \\
\hline 15 & Hand Breadth. & $8.2 \pm 1.2$ & 28.8 & $\mathrm{p}<0.0001 \mathrm{HS}$ & 7.1 & 7.6 & 10.4 \\
\hline \multicolumn{2}{|c|}{ Wrist-centre of grip length. } & $8.1 \pm 0.7$ & 45.4 & $\mathrm{p}<0.0001 \mathrm{HS}$ & 6.2 & 8.5 & 8.7 \\
\hline \multicolumn{2}{|c|}{ Forward bending of the head. $\left(<20^{\circ}\right)$} & $16.8 \pm 0.8$ & 32.9 & \multicolumn{4}{|c|}{$\mathrm{p}<0.0001 \mathrm{HS}$} \\
\hline \multicolumn{2}{|c|}{ Trunk rotation. $\left(<20^{\circ}\right)$} & $17.04 \pm 1.6$ & 15.9 & \multicolumn{4}{|c|}{$\mathrm{p}<0.0001 \mathrm{HS}$} \\
\hline \multicolumn{2}{|c|}{ Trunk sideward inclination. $\left(<10^{\circ}\right)$} & $7.3 \pm 2.1$ & 16.9 & \multicolumn{4}{|c|}{$\mathrm{p}<0.0001 \mathrm{HS}$} \\
\hline \multicolumn{2}{|c|}{$\operatorname{BMI}\left(\mathrm{kg} / \mathrm{m}^{2}\right)$} & $25.0 \pm 0.6$ & 27.8 & \multicolumn{4}{|c|}{$\mathrm{p}<0.0001 \mathrm{HS}$} \\
\hline
\end{tabular}

Table 4: Anthropometric Measurement-Male (N=44) Statistical Inference: HS-Highly significant SS- Statistical significant.

\begin{tabular}{|c|c|c|c|c|c|c|c|}
\hline \multirow{2}{*}{ Ref no } & \multirow{2}{*}{ Variables } & \multirow{2}{*}{ Mean \pm SD } & \multirow{2}{*}{$Z$ value } & \multirow{2}{*}{ p-value } & \multicolumn{3}{|c|}{ Percentile } \\
\hline & & & & & $5^{\text {th }}$ & $50^{\text {th }}$ & $95^{\text {th }}$ \\
\hline 1 & Stature. & $169.2 \pm 2.9$ & -45.6 & $\mathrm{p}<0.0001 \mathrm{HS}$ & 165 & 170 & 173 \\
\hline 2 & Sitting Height. & $75.9 \pm 1.6$ & 23.9 & $\mathrm{p}<0.0001 \mathrm{HS}$ & 74.1 & 75.1 & 79.9 \\
\hline 3 & Sitting Eye Height. & $77.7 \pm 2.1$ & -19.2 & $\mathrm{p}<0.0001 \mathrm{HS}$ & 74.3 & 78.9 & 80.6 \\
\hline 4 & Sitting Shoulder Height. & $60.7 \pm 2.1$ & 11.5 & $\mathrm{p}<0.0001 \mathrm{HS}$ & 59.1 & 59.7 & 66.8 \\
\hline 5 & Sitting Elbow height. & $17.0 \pm 3.3$ & 5.6 & $\mathrm{p}<0.0001 \mathrm{HS}$ & 12.1 & 17.0 & 22.2 \\
\hline 9 & Knee Height. & $47.7 \pm 4.9$ & -2.3 & $\mathrm{p}=0.17 \mathrm{SS}$ & 40.6 & 47.8 & 55.1 \\
\hline 12 & Shoulder-Elbow Length. & $30.2 \pm 1.5$ & 3.6 & $\mathrm{p}<0.0001 \mathrm{HS}$ & 28.1 & 30.3 & 32.5 \\
\hline 13 & Elbow Fingertip Length. & $51.5 \pm 5.8$ & 1.2 & $\mathrm{p}<0.0001 \mathrm{HS}$ & 39.7 & 54.8 & 57.2 \\
\hline 14 & Hand length. & $17.5 \pm 1.5$ & 12.1 & $\mathrm{p}<0.0001 \mathrm{HS}$ & 14.9 & 17.6 & 19.4 \\
\hline 15 & Hand Breadth. & $7.6 \pm 0.9$ & 30.9 & $\mathrm{p}<0.0001 \mathrm{HS}$ & 5.2 & 7.7 & 8.8 \\
\hline \multicolumn{2}{|c|}{ Wrist-centre of grip length. } & $6.7 \pm 1.1$ & 25.6 & $\mathrm{p}<0.0001 \mathrm{HS}$ & 6.6 & 7.1 & 8.3 \\
\hline \multicolumn{2}{|c|}{ Forward bending of the head. $\left(<20^{\circ}\right)$} & $15.9 \pm 0.4$ & 14.0 & \multicolumn{4}{|l|}{$\mathrm{p}<0.0001 \mathrm{HS}$} \\
\hline \multicolumn{2}{|c|}{ Trunk rotation. $\left(<20^{\circ}\right)$} & $16.4 \pm 1.7$ & 11.0 & \multicolumn{4}{|l|}{$\mathrm{p}<0.0001 \mathrm{HS}$} \\
\hline \multicolumn{2}{|c|}{ Trunk sideward inclination. $\left(<10^{\circ}\right)$} & $5.7 \pm 2.8$ & 13.5 & \multicolumn{4}{|l|}{$\mathrm{p}<0.0001 \mathrm{HS}$} \\
\hline \multicolumn{2}{|c|}{$\mathrm{BMI}\left(\mathrm{kg} / \mathrm{m}^{2}\right)$} & $22.9 \pm 1.4$ & 8.5 & \multicolumn{4}{|l|}{$\mathrm{p}<0.0001 \mathrm{HS}$} \\
\hline
\end{tabular}

Table 5: Anthropometric Measurement-Female $(n=36)$ Statistical Inference: HS-Highly significant $\quad$ SS- Statistical significant. 


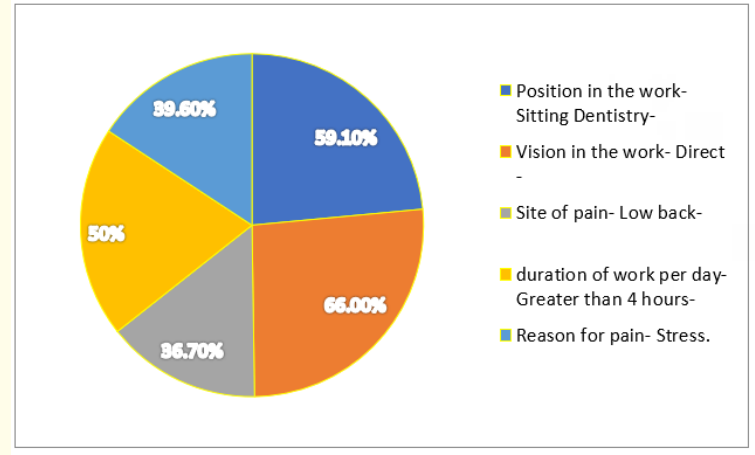

Graph 3: Standardized Nordic Questionnaire- Dental (n=174).

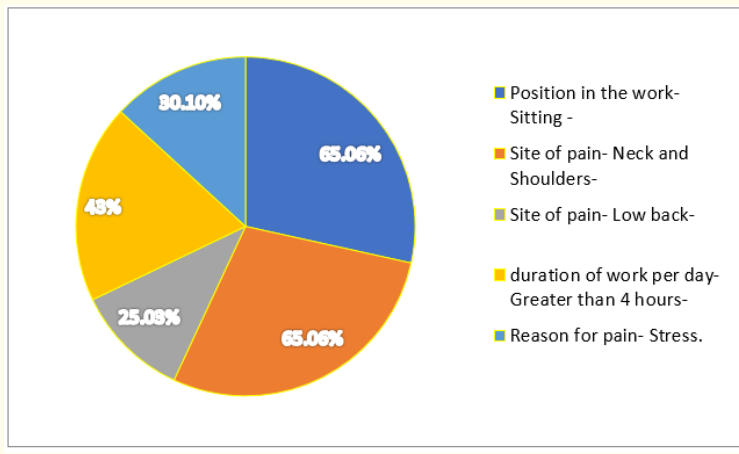

Graph 4: Standardized Nordic Questionnaire- Medical (n=83).

\section{Discussion}

All published studies on musculoskeletal problems have an observational design. Using questionnaires, as in our cases which are useful for identifying the prevalence of a disorder, determining the clinical features of patients, and for designing possible preventive strategies [16].

Our study found that most professionals referred to some musculoskeletal pain in the last six months73.5\% ( $\mathrm{n}=189)$ in coincidence with the survey done by Szymańska J [17].

The role of age is even more controversial. While there are studies that claim that the frequency of pain remains stable with age, others believe that musculoskeletal discomfort is maximum around the sixth decade of life [18].
In our study, we found young professionals to have a higher incidence of low backache, followed by neck pain due to incorrect working positions which was in accordance to the study by Paknahad Z., et al. [19] Certain factors help the participants to relieve their pain which includes analgesic drugs (30\%), muscle relaxing exercise $(26.4 \%)$, correct posture $(17.8 \%)$, and pause for few minutes $(12.4 \%)$, seek specialist opinion (10.5\%) etc. Similar to this study, a study in Glasgow also found that improving or correcting posture can definitely help to relieve the pain [20].

All the percentile values indicate that the sample are longlegged, which was in par with the present study. Kanteshwari., et al. [21] and Gupta., et al. [22] indicated only 50\% of the respondents were aware of Ergonomics and 59.6\% in another study done by Gopinadh., et al. [23].

In a recent study, Park., et al. [24] defined the low back and neck as the riskiest regions of the body for the Dentists developing MSD. In our research, lower back pain was the most prevalent complaint, followed by neck and shoulder pain.

Lindfors., et al. [25] in 2006 reported that female group of Dentists showed a higher incidence of muscular pain, contradictory to this, our study showed no significant difference in the presence of pain among males and females.

Body Mass Index (BMI) is a critical Anthropometric index that is commonly used for body fat storage status assessment and is used for body fat determination [26].

According to Pheasant and Haslegrave [20], Body Mass Index (BMI) is a critical anthropometric index that is commonly used for body fat storage status assessment and used for body fat determination.

Frisancho [27] suggests that BMI within the ranges of 18.50 $25.00 \mathrm{~kg} / \mathrm{m} 2$ considered as usual. In this study, the mean of BMI is $25.0 \pm 0.6 \mathrm{~kg} / \mathrm{m}^{2}$ for male's and $22.9 \pm 1.4 \mathrm{~kg} / \mathrm{m}^{2}$ for females indicating that the subjects were normal (healthy weight).

\section{Recommendations}

a) More extensive longitudinal studies using a larger sample size which includes a physical examination.

b) Further studies to identify the major-specific risk factors related to MSD and how to prevent them 
c) Studies regarding the Dentists' and Medical professionals, knowledge regarding MSD and ergonomics and increasing their awareness for a longer and healthier career.

\section{Limitations of this study are as follows}

a) After regions at risk identified through questionnaires, no Ergonomic intervention were undertaken; however, Ergonomic training was planned to be delivered.

b) Functional limitations and quality of life not evaluated.

c) Despite the efforts made to increase the sample size, the final sample was smaller than desired.

d) The severity of the symptoms, rest breaks and number of days on which the professionals could work not inquired

e) The models achieved in this study were based on an adult sample and did not apply to juveniles.

\section{Conclusion}

a) The prevalence of MSD among Dentists was high when compared to the Medical profession.

b) Labor gymnastics associated with Ergonomic principles can minimize to the professionals health disorders caused by occupational activities, improving their quality of life.

c) Good working Ergonomics is essential so that work capability, efficiency, and high clinical level of treatment maintained throughout the working life.

d) Lower back pain proved to have the highest prevalence followed by Neck and shoulders

e) There was no significant relationship between years of practicing (years of experience) and MSD.

\section{Ethical approval}

non-invasive study.

\section{Ethical disclosures}

- Protection of human and animal subjects: The authors declare that no experiments performed on humans or animals for this study.

- Confidentiality of data: The authors declare that no patient data appear in this article.

- $\quad$ Right to privacy and informed consent: The authors have obtained the written informed consent of the patients or subjects mentioned in the article. The corresponding author owns this document.

\section{Conflict of interest and source of funding}

The author declares that there is no exceptional financial support for this research work from the funding agency and there is no conflict of interest among all authors

\section{Acknowledgment}

All the authors express sincere gratitude to all respondents whose honest attention help and support, and the participants of the study lead the Research project to a worthful outcome.

\section{Bibliography}

1. Ergonomics design and analysis TM. Dassault Systemes France (2002).

2. Da Costa and Vieira. "Risk factors for work-related musculoskeletal disorders: A systematic review of recent longitudinal studies". American Journal of Industrial Medicine 53.3 (2010): 285-323.

3. Scully C., et al. "Mortality and some aspects of morbidity". In: Occupational Hazards to Dental Staff British Medical Journal, London (1990): 1-21.

4. Burke FJI and Main JR. "The Practice of Dentistry: An assessment of reasons for premature retirement". British Dental Journal 182.7 (1997): 250-254.

5. Movahhed T., et al. "Do dental students have a neutral body posture?". Journal of Back and Musculoskeletal Rehabilitation 29 (2016): 859-864.

6. Valachi B and Valachi K. "Mechanisms leading to musculoskeletal disorders in dentistry". Journal of American Dental Association 134.10 (2003):1344-1350.

7. Americal Dental Association INFO Pak. "Ergonomics for Dental Students". Ada Info Pak (2008): 1-4.

8. Russell JG. "Ergonomics in the Dental Surgery". Occupational Medicine 23 (1973):128-131.

9. Pekka Kahri. "Ergonomics and teamwork in dental treatment". Planmeca Oy 02 (2005): 1-2.

10. Leggat PA, Smith DR. "Musculoskeletal disorder self reported by dentists in Queensland, Australia". Australia Dental Journal 51.4 (2006): 324-327.

11. SZM Dawal., et al. "Anthropometric database for the learning environment of high school and university students". International Journal of Occupational Safety and Ergonomics 18.4 (2012): 461-472. 
12. J Majumder. "Anthropometric dimensions among Indian males-a principal-component analysis". Eurasian Journal of Anthropology 5.2 (2014): 54-62.

13. JJ Shiru and S Abubakar. "Anthropometry in engineering design (a case study of cassava grating machines installed in Doko and Kutigi metropolis of Lavun local government areas of Niger state)". Nige Acad Forum 22.1 (2012) 132-139.

14. Kuorinka., et al. "Standardised Nordic questionnaires for the analysis of musculoskeletal symptoms". Applied Ergonomics 18.3 (1987): 233-237.

15. Stephen Pheasant Christine M. Haslegrave. "Body space: Anthropometry, Ergonomics and the Design of Work". 3rd Edition (2005).

16. Lalumandier JA., et al. "Musculoskeletal pain: prevalence, prevention, and differences among dental office personnel". General Dentistry 49 (2001): 160-166.

17. Szymańska J. "Disorders of the musculoskeletal system among dentists from the aspect of ergonomics and prophylaxis". Annals of Agricultural and Environmental Medicine 9 (2002): 169-173.

18. Shrestha BP., et al. "Work-Related Complaints among Dentists". Journal of Nepal Medical Association 47 (2008): 77-81.

19. Paknahad Z., et al. "Body mass index and its relationship with hematological indices in Iranian women". Pakistan Journal of Nutrition 7.2 (2008): 377-380.

20. S Pheasant and CM Haslegrave Body Space: "Anthropometry, Ergonomics and the Design of Work", CRC Press, 3rd edition (2005).

21. Kanteshwari K., et al. "Correlation of awareness and practice of working postures with the prevalence of musculoskeletal disorders among dental professionals". General Dentistry 59 (2011): 476-483.

22. Gupta AA., et al. "Ergonomic microscope: Need of the hour". Journal of Clinical and Diagnostic Research 9 (2015): ZC62ZC65.

23. Gopinadh A., et al. "Ergonomics and musculoskeletal disorder: As an occupational hazard in dentistry". The Journal of Contemporary Dental Practice 14 (2013): 299-303.
24. Park HS., et al. "Analysis of the risk factors of musculoskeletal disease among dentists induced by work posture". The Journal of Physical Therapy Science 27 (2015): 3651-3654.

25. Lindfors P., et al. "Work characteristics and upper extremity disorders in female dental health" 48 (2006): 192-197.

26. Okosun IS., et al. "Abdominal adiposity in six populations of West Africa descent: prevalence and population attributable fraction of hypertension". Obesity Research 7.5 (1999): 45344562 .

27. R Frisancho. "Anthropometric Standards for the Assessment of Growth and Nutritional Status". University of Michigan Press (1990).

\section{Volume 3 Issue 11 November 2019}

(C) All rights are reserved by K Srinivasan., et al. 